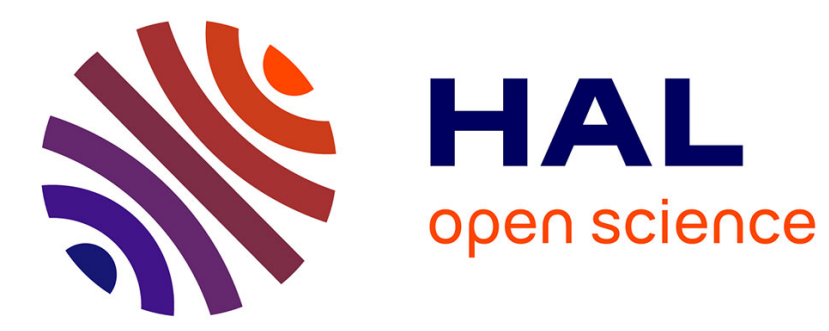

\title{
Comprehensive Pre-Clinical Evaluation of a Multi-physics Model of Liver Tumor Radiofrequency Ablation
}

Chloé Audigier, Tommaso Mansi, Hervé Delingette, Saikiran Rapaka, Tiziano Passerini, Viorel Mihalef, Marie-Pierre Jolly, Raoul Pop, Michele Diana, Luc Soler, et al.

\section{To cite this version:}

Chloé Audigier, Tommaso Mansi, Hervé Delingette, Saikiran Rapaka, Tiziano Passerini, et al.. Comprehensive Pre-Clinical Evaluation of a Multi-physics Model of Liver Tumor Radiofrequency Ablation. International Journal of Computer Assisted Radiology and Surgery, 2016, 10.1007/s11548-016-1517x . hal-01423321

\section{HAL Id: hal-01423321 \\ https://hal.inria.fr/hal-01423321}

Submitted on 29 Dec 2016

HAL is a multi-disciplinary open access archive for the deposit and dissemination of scientific research documents, whether they are published or not. The documents may come from teaching and research institutions in France or abroad, or from public or private research centers.
L'archive ouverte pluridisciplinaire HAL, est destinée au dépôt et à la diffusion de documents scientifiques de niveau recherche, publiés ou non, émanant des établissements d'enseignement et de recherche français ou étrangers, des laboratoires publics ou privés. 


\title{
Comprehensive Pre-Clinical Evaluation of a Multi-physics Model of Liver Tumor Radiofrequency Ablation
}

\author{
Chloé Audigier ${ }^{1,2}$. Tommaso Mansi ${ }^{2}$. Hervé Delingette ${ }^{1}$. \\ Saikiran Rapaka ${ }^{2}$. Tiziano Passerini ${ }^{2}$. Viorel Mihalef ${ }^{2}$. \\ Marie-Pierre Jolly ${ }^{2}$. Raoul Pop ${ }^{3}$. Michele Diana ${ }^{3}$. Luc Soler ${ }^{3,4}$. \\ Ali Kamen ${ }^{2}$. Dorin Comaniciu ${ }^{2}$. Nicholas Ayache ${ }^{1}$
}

Received: Aug 04, 2016 / Accepted: Dec 21, 2016

\begin{abstract}
Purpose We aim at developing a framework for the validation of a subject-specific multi-physics model of liver tumor radiofrequency ablation (RFA).

Methods The RFA computation becomes subject-specific after several levels of personalization: geometrical and biophysical (hemodynamics, heat transfer and an extended cellular necrosis model). We present a comprehensive experimental set-up combining multi-modal, preand post-operative anatomical and functional images, as well as the interventional monitoring of intra-operative signals: the temperature and delivered power.

Results To exploit this data set, an efficient processing pipeline is introduced, which copes with image noise, variable resolution and anisotropy. The validation study includes twelve ablations from five healthy pig livers: a mean point-to-mesh error between predicted and actual ablation extent of $5.3 \pm 3.6 \mathrm{~mm}$ is achieved.

Conclusion This enables an end-to-end pre-clinical validation framework that considers the available data set.
\end{abstract}

Keywords Computational Modeling · Radiofrequency Ablation · Pre-clinical Evaluation

Chloé Audigier

2004 route des Lucioles BP-93, 06902 Sophia-Antipolis Cedex, FRANCE

Tel. : +33492387926

E-mail: chloe.audigier@inria.fr

1 Université Côte d'Azur and Inria Sophia-Antipolis Méditerranée Asclepios team, Inria Sophia Antipolis

2 Medical Imaging Technologies, Siemens Healthcare, Princeton, NJ, USA

${ }^{3} \mathrm{IHU}$ - Institut de Chirugie Guidée par l'Image, Strasbourg, France

${ }^{4}$ IRCAD - Institut de Recherche contre les Cancers de l'Appareil Digestif, Strasbourg, France

\section{Introduction}

During liver tumor radiofrequency ablation (RFA), the clinician inserts a probe percutaneously or during open surgery in the liver parenchyma, visualization of the thermal induced necrosis progress is then unavailable. Tailoring the heating protocol to the individual patient is thus challenging due to inter-subject variability in tissue characteristics [1], the heterogeneous cooling effect of large neighboring vessels [2], porous circulation [3] and blood coagulation [4]. As a result, subjectspecific computational modelling of RFA could improve the planning of the procedure and provide additional guidance during the intervention for a given patient. More specifically, such models could potentially estimate in-vivo temperatures, calculate the size, the shape and the location of the necrotic area, given the position and settings of the ablation probe in the liver. Thus they could help the clinician in deciding where to place the heating probe and for how long heating must be applied in order to fully ablate the lesion. For instance, the probe placement has been optimized in [5] by using segmentation masks and the exploration of the set of pareto-efficient solutions.

Several approaches have been developed to describe and model RFA of liver tumors. They differ in their choice of the biophysical phenomena considered and the type of experimental data used to design and validate them. All simulations are based on the bioheat equation considering a cooling effect that is either neglected [6], spread all over the liver [4] or considering the hepatic vasculature [6-9]. The bioheat equation is simulated based on the most common discretization method: the Finite Element Method (FEM) $[6,7,10]$ or the Lattice Boltzmann Method (LBM) [9,11]. Other authors propose a simplified model using a weighted 
distance-based method $[5,12]$ to approximate the ablation zone in real-time using graphics processing units (GPU), but the analysis of the effect of an approximation instead of the complex numerical simulation has not been done yet. The cooling effect due to venous flow in the parenchyma is also considered in $[8$, 9 ], whereas it is not the case for arterial flow. However hepatic veins, portal vein and the hepatic arteries have significantly different effects on the thermalinduced lesion [13], which might be due to the difference in their flow velocities, profile and microvascular branching pattern. While the Pennes model [14] accounts for parenchymal perfusion in a simplified manner, by assuming a uniform distribution throughout the tissue, a more comprehensive model is needed to evaluate its effect. Hepatic perfusion can be simulated precisely based on micro-CT [15], but we propose an elaborated model based on vessels segmented from CT images, which does not require mesh generation, simplify the setting of boundary conditions and reduce the computational time compared to Darcy flow model for example [9].

Few authors [7-9] propose to simulate RFA on realistic subject-specific geometries extracted from images and only [11] has personalized biophysical parameters on patient data to minimize the discrepancy between simulated and measured necrotic regions. Indeed, parameters in the literature often come from tissue experiments on different species ex-vivo [1], and their application to human is not granted.

Up to now, the comparison between simulated and measured necrotic regions has been used by several authors [7-9] as the main criteria of success in calculating the effect of RFA on abdominal tumors, for either model validation or personalization. However, the necrosis of tissue is the resultant of several combined physical phenomena, mainly the heat transfer and cell death mechanisms, meaning that a given ablated region may be explained by several combinations of parameters. In addition to this identifiability issue, the size of the tumor extent can only be known reliably from post-operative imaging and its shape may be highly asymmetric [1], which makes it difficult to eventually update the ablation plan during the procedure. A method that relies also on pre-operative or interventional data for validation or personalization is therefore required for RFA models to be clinically useful. Those observations are complementary information to the necrotic extent for model evaluation.

Computational models of RFA depend on a large number of different parameters, which depend mostly on the patient, the temperature or the current state of the tissue. They also have a high computationnal cost.
Understanding the observability of parameters is challenging with clinical data but is more feasible on preclinical data in a more controlled environment. This extensive validation step is important prior to any translation into clinical settings. It is paramount because the size and the location of the RFA lesion has to be predictable and controllable for clinical applications. A comprehensive understanding of detailed multi-physics model is required first to simplify only the aspects that are not needed, which could then enable model simplification and personalization, and eventually computational models of RFA can be helpful for interventional guidance or therapy planning.

In this paper, a pre-clinical study for validation of RFA model is introduced, based on pre-, intra- and post-operative data (Sec. 2). We first present the data analysis required before using the information from the images into the model. Since our approach relies on pre-clinical data of healthy pigs in a controlled environment, the validation leads to an increased confidence in the computed information.

The computational model, implemented using LBM, is personalized at different levels: the anatomy is estimated from Computed Tomography (CT) and blood flow from Phase-Contrast MR imaging and invasive measurements (Sec. 3). Heat transfer is computed according to the bioheat equation, coupled with a novel cellular necrosis model, equivalent to the one proposed in [16], except that we do not assume a single forward rate coefficient. A Computational Fluid Dynamics (CFD) solver which incorporates a porous part to deal with the liver parenchyma is also used. In Sec. 4, an evaluation of each pre-processing step is performed first to highlight the different sources of error. The model is evaluated on twelve ablations from five healthy pigs, inside which surrogate tumors have been implanted. Based on postoperative images and measurements, we present suitable predictions of necrotic region extent, temperature and power evolution. As we rely on a LBM implementation on GPU, a single simulation is faster than real time, which allows us to adjust key biophysical parameters. Those key model parameters (heat conductivity and heat capacity) are estimated from this pipeline by minimizing the error between the computed power and cooling temperature and the observed values. Sec. 6 concludes the paper.

This study extends our previous work [9] on a clinical dataset of 10 patients. In this work, a novel cell death model is proposed and the CFD solver used to compute the blood flow is also new. We use a complete patient-specific geometry including hepatic venous and arterial circulation systems, the addition of patientspecific boundary conditions acquired pre-operatively 
by imaging and invasive measurements. The estimation of the main parameters of the model is performed automatically and the evaluation of the proposed model is performed on a pre-clinical dataset of five pigs from a comprehensive experimental set-up specially designed for RFA model validation.

\section{Pre-clinical Data Acquisition \& Processing}

A comprehensive animal experiment has been realized specifically for model validation and parameter identification. It includes several modalities at pre-, intra- and post-operative stages. Fig. 1 illustrates the available pre-clinical data (in blue), the different pre-processing steps needed (in red, green and white) before performing the computation (in grey).

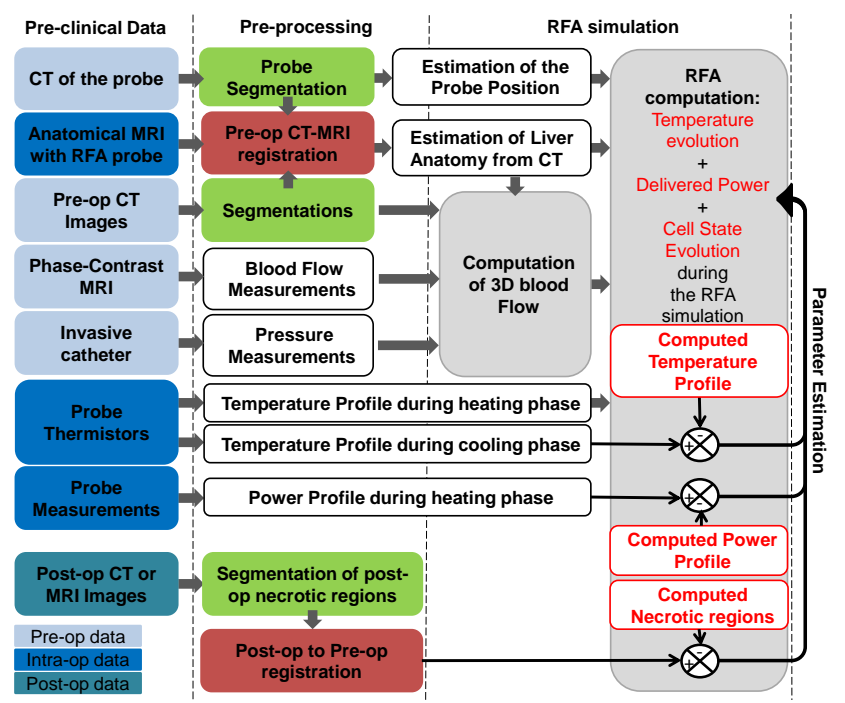

Fig. 1 Pipeline presenting the available pre-clinical data in blue, the pre-processing needed to use those data (segmentation in green, registration in red), the different computations done and finally a possible personalization framework.

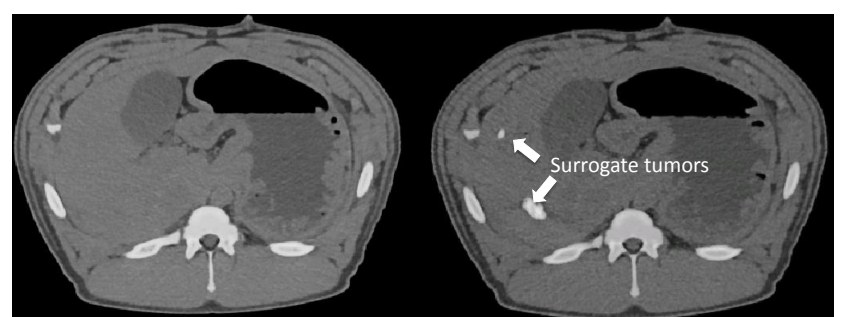

Fig. 2 Two CT images of Pig 1. (Left) Before surrogate tumor implantation; (Right) After surrogate tumor implantation.

\subsection{Experimental Set-up}

The present experimental study (No. 38.2014.01.063) received approval from the local Ethical Committee on Animal Experimentation. All animals used in the experimental laboratory were managed according to French laws for animal use and care and according to the directives of the European Community Council (2010/63/EU).

Pigs are considered as a relevant animal model as their hepatic system is similar to the human one. Several surrogate tumors (diameters $<3 \mathrm{~cm}$ ) are inserted on five swines to mimic the clinical workflow, where the clinician has to insert the RFA probe targeting the tumor and to choose its diameter depending on the tumor size. They are implanted at various locations of the liver (close to vessels or the Glisson capsule) under ultrasound (US) guidance (SIEMENS ACUSON S3000), followed by the acquisition of pre-operative CT images including portal, venous and arterial phases (SIEMENS Somatom Definition A5) since it has been showed that the large vessels play an important role in the shape of the necrotic lesion [9]. The surrogate tumors are made of a specific gel (a mix of biocompatible gelatin, alginate and nanoparticles) which exhibits an hyper-intense signal in CT and MRI as illustrated in Fig. 2. We refer to the different tumors as Pig $\mathrm{n}-\mathrm{m}$, for the $m^{\text {th }}$ tumor inserted in the $n^{t h}$ pig. A catheter is introduced through the jugular vein to get the free and the wedge pressure in a subhepatic vein and in the vena cava. An MR-compatible RFA probe, the radiofrequency interstitial tumor ablation (RITA) probe (StarBurst RFA, AngioDynamics; www.angiodynamics.com), is deployed at $2 \mathrm{~cm}$ of diameter (the diameter of the area defined by the tips of the probe is $2 \mathrm{~cm}$ ) under US guidance (Fig. 3) next to the targeted surrogate tumor. An MR image is then acquired to get the position of the probe in the liver (SIEMENS Magnetom Aera 1.5T) and flow data. The temperature and delivered power are monitored (there are thermistors at the probe tips) and recorded intra-operatively during and after the ablation. Finally, to assess the extent of the necrotic areas, a post-operative $\mathrm{CT}$ without contrast agent to limit the radiation exposure for ethical reason), $\mathrm{T} 2$ or $\mathrm{T} 1+$ gadolinium MRI are acquired two days after the ablation [17-19]. Unfortunately, all the post-operative images were not systematically acquired. We would acquire as many images as possible, but depending on the pig condition, and/or the procedure duration (individual experimental changes), we were not able to acquire all the images in every case (see supplementary materials for available images). Overall, pre-, intra- and post-operative images are available, along with interventional device measurements (Fig. 1). To the best of 


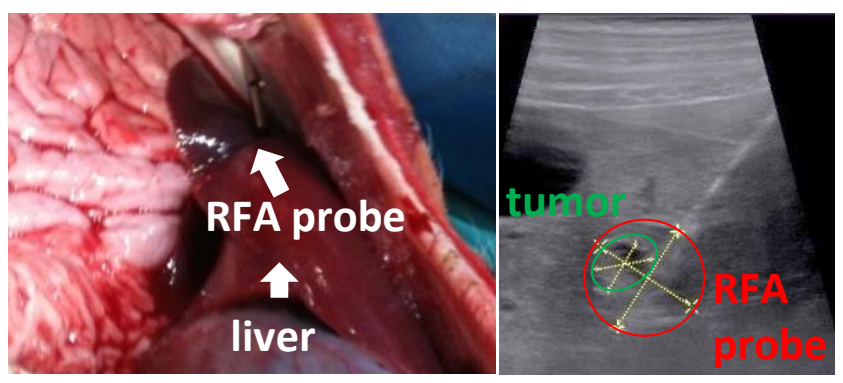

Fig. 3 (Left) Picture of the probe inside the pig liver; (Right) U.S image of the probe next to the surrogate tumor. The probe diameter is shown in red, the tumor diameter in green.

our knowledge, no such validation set-up has been reported previously in the literature.

\subsection{D Modeling of the RFA Probe}

The geometry of the probe deployed at $2 \mathrm{~cm}$ is acquired from a CT image (resolution: $0.2 \times 0.2 \times 0.9 \mathrm{~mm}$ ) of the probe alone. A $3 \mathrm{D}$ mesh is then reconstructed by thresholding the image intensity (Fig. 4, left), and manually registered to the pre-operative CT using the main axis of the probe and intra-operative MR data. The manual rigid registration is done using Paraview ${ }^{1}$ and is visually checked by an expert up to a rotation along the probe axis. While the main axis is clearly visible as well as three or four tips (depending on the ablation considered), the MR resolution does not allow to distinguish between the nine tips of the probe individually (Fig. 4, right).
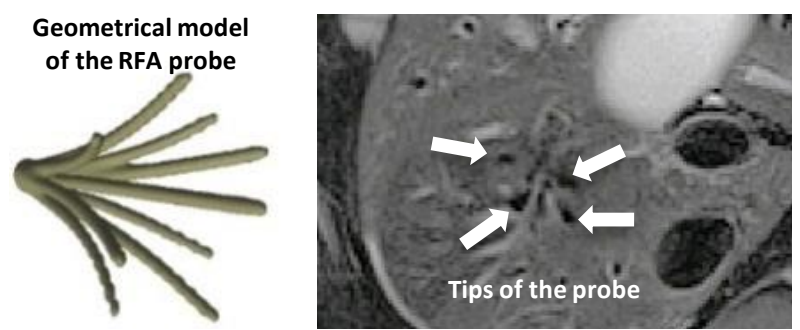

Fig. 4 (Left) Mesh model of the probe with the 9 tips derived from a CT image of the probe only; (Right) Anatomical MRI with the MRI-compatible probe implanted in the liver. Four tips are visible.

\subsection{Data Pre-processing: Segmentation}

As each structure of interest is needed as input of the RFA computational model, the segmentation of patient

\footnotetext{
1 http://www.paraview.org/
}

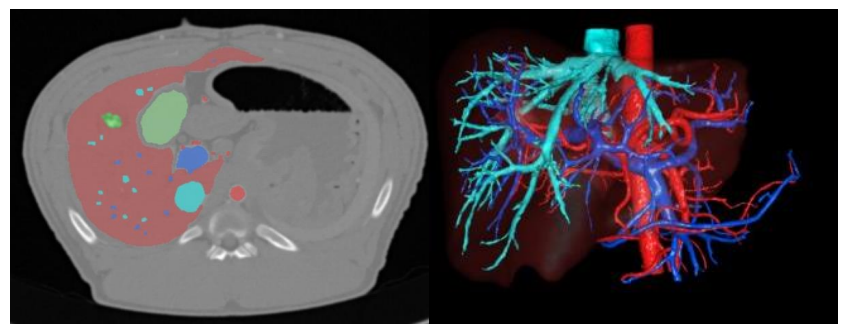

Fig. 5 Segmentation of the liver in magenta, arterial vessels in red, portal and hepatic networks in light blue and dark blue respectively, surrogate tumors in dark green and gall bladder in light green. (Left) Overlay of the segmented areas on preoperative CT image. (Right) Vessels and liver parenchyma.

images is a determinant task. From the pre-operative CT data, the following anatomical and pathological regions are segmented semi-automatically and meshed by the tools of Visible Patient, Strasbourg, France [20] (Fig. 5): parenchyma, hepatic veins, vena cava, portal vein, hepatic arteries and all tumors. These regions are then used to define the computational domain. The meshes are rasterized and a multi-label mask image is created to identify the different structures on a Cartesian grid of user-defined resolution. To define the computational domain, a level set representation of the liver without tumor and vessels is computed. Necrotic areas around the surrogate tumor gel are segmented and meshed from the available post-operative CT or MR data on which it is the most visible.

\subsection{Data Pre-processing: CFD Computational Domain}

Smooth Vessel Trees. The pre-operative images from which the vessels are segmented have anisotropic resolution. To avoid unstable solution of the CFD solver, smooth vessel trees have to be generated from the semiautomatic segmentations. To this end, centerlines are extracted from each vessel segmentation using VMTK ${ }^{2}$ (Fig. 6, left). Along each branch of the centerline, the mean radius is computed and the smooth vessel tree is generated by overlaying cylinders having this mean radius in a piecewise fashion (Fig. 6, right). All those cylinders are rasterized on a single image.

Blood flow from Phase-Contrast MRI. The computational model of RFA requires as inputs the blood flow entering the vena cava, the portal vein and the hepatic artery. Instead of fixing nominal values from the literature, $2 \mathrm{D}+\mathrm{t}$ Phase-Contrast MR images were acquired pre-operatively before the probe implantation in order to impose subject-specific values as boundary conditions.

2 The Vascular Modeling Toolkit, www.vmtk.org. 


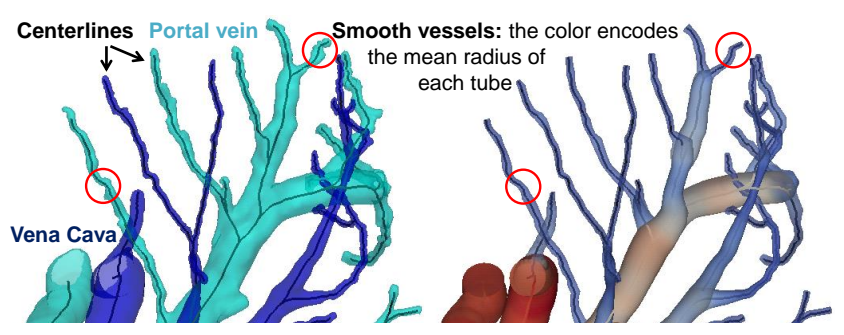

Fig. 6 (Left) Centerlines extraction from the vessel segmentation. The segmentation gives non-smooth vessels due to the anisotropy of input images. (Right) Smooth vessels generated from the centerlines.

For each pig, several 2D+time sequences are acquired at different time points (9 sequences on average, min: 3, max: 17), for the reproducibility of data, and also at different locations: at the inlet of the hepatic artery, at the inlet of the portal vein and at the inlet of the vena cava. Given one $2 \mathrm{D}+\mathrm{t}$ sequence, the user places a single seed in the vessel of interest in the first image and then an automatic method is used to segment this vessel on each $2 \mathrm{D}$ slice (Fig. 7, right) [21, $22]$. Using the vessel area defined by the segmentation, the mean blood flow can be computed at each time. If $\mathrm{N} 2 \mathrm{D}+\mathrm{t}$ sequences are acquired at the same location but at different time points, this is done $\mathrm{N}$ times and the RMS of the mean of those $\mathrm{N}$ curves is used. As we learned throughout the cases how to best acquire data, we decided to use the measurements of Pig 5 for all the other cases to cope with noise, non-reproducible and non-coherent measures on the first four pigs. We assume that the values will not vary much, which is reasonable since all the pigs were healthy, of similar weight and age. For Pig 5 (vena cava), the acquisition has been done at 3 different time points $(\mathrm{N}=3)$. The RMS of the mean of those three curves is used (Fig. 7, left black curve) since numerical results have shown that pulsatile velocity profile in large blood vessels has little difference in effect on the thermal lesion region of tissue compared with uniform or parabolic velocity profile [23].

Pressure from Invasive Measurements. The computational model of RFA requires also vena cava and hepatic artery blood pressures as inputs (See arrows on Fig. 9 for the locations where the boundary conditions are applied). In order to impose subject-specific values as boundary conditions, these blood pressures have been measured to avoid the use of nominal values from the literature. The pressures at the outlet of the vena cava and the hepatic artery were measured invasively by catheter introduced through the jugular vein. The same values (from Pig 5) are used as boundary conditions in the five pigs, as it is the case for the blood flow measurements.

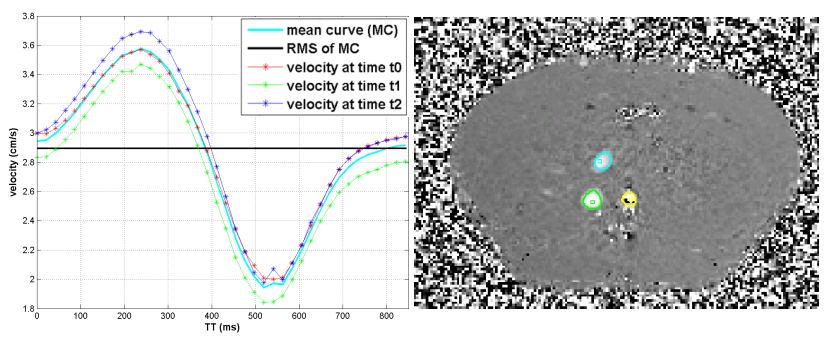

Fig. 7 (Left) Velocity in the vena cava of Pig 5 from PhaseContrast MRI with respect to trigger time (all measures are registered on the cardiac pulse). In cyan, the mean of different measurements at different times (red, blue, green curves). The RMS of this curve (black curve) is then used as boundary conditions in the computation as $\varphi_{\text {vcin }}$. (Right) Phase-Contrast MRI of Pig 5: the vena cava is segmented in green, the portal vein in blue and the aorta in yellow.

Porosity Map. The porosity, defined as the fraction of blood volume $\left(V_{b}\right)$ over the total volume $\left(V_{t}\right): \epsilon=V_{b} / V_{t}$, has to be defined everywhere in the computational domain as it is an input of the CFD solver. The vessel walls are defined as follow. The smooth vessel trees are rasterized on a single image. On this image, we perform a 26-connexity dilation on the voxels of the vessels to model the endothelium and avoid the flow to go through the vessel wall. The extremities are detected using the centerlines previously used and the porosity at the extremities is set to the parenchyma porosity value. Fig. 8 shows an example of porosity map. The porosity value is 1 in the CT-visible vessels, 0.1 in the parenchyma [8], modeled then as a porous medium. Experiments have been performed to obtain a sufficiently small porosity (0.04) in the vessel walls (impermeable medium) to avoid the occurrence of shear stress on the vessel walls (leakage) [11].

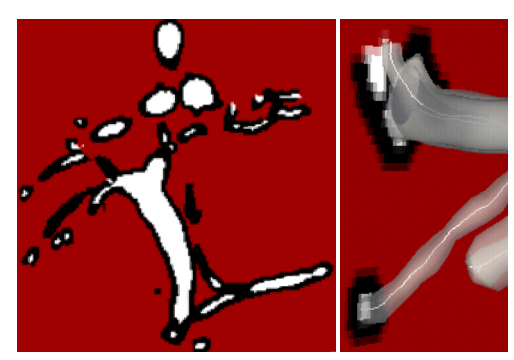

Fig. 8 (Left) The porosity map of Pig 1 created from the vessels segmentation. The porosity is 1 in the vessels (in white), 0.04 in the endothelium (in black) and 0.1 in the parenchyma. (Right) Zoom on the porosity map. The extremities of the vessels are detected using the centerlines so that the blood flow can go through the vessel. 


\subsection{Data Pre-processing: Post-To-Pre Registration}

Due to ethical reasons, CT with contrast agent could not be acquired two days after intervention, making accurate post- to pre-operative registration challenging. In order to compare the results of the computation with the ground truth given by the post-operative images, registration of the post- to the pre-operative image is performed. For each pig, the pre-operative image (CT from different phases) does not necessarily belong to the same modality as the post-operative image (CT or MRI (T2 or T1 + gad)) due to individual experimental changes. Moreover, the pigs are neither in a similar position nor a similar condition, as they had an empty stomach the day of the intervention, which was not true two days after. Breathing has also an important impact on the shape of the liver. For those reasons, we choose to register the post-operative meshes to the pre-operative image instead of pure image-to-image registration. From the semi-automatic segmentation of the post-operative image, a tetrahedral mesh is generated using CGAL ${ }^{3}$. From the segmentation of the pre-operative image, a binary mask of the liver including vessels and arteries is generated. Using the SOFA framework ${ }^{4}$, the post-operative mesh is deformed elastically to match the intensity profile in the pre-operative image using the Finite Element Method (FEM) and a co-rotational model [24]. First-order implicit Euler time integration is employed and the system of equation is solved with the conjugate gradient algorithm. The computed deformation field is then applied to the necrotic surface mesh using a barycentric mapping between the coordinates of the surface mesh and the tetrahedral mesh (12 000 tetrahedra and 3000 nodes for Pig 5). The registration is fast to perform, it took 178 seconds on a Windows 7 laptop machine (Intel Core, $2.40 \mathrm{GHz}$, 8GB RAM, 8 CPUs) on average to register the postoperative mesh to the pre-operative image.

\section{Subject-Specific RFA Model}

\subsection{Liver Blood Flow Computation}

Model Description. We want to get simultaneously the blood flow in the large visible venous and arterial vessels, as well as in the parenchyma. To that end, the flow in the main vessels and in the parenchyma is simultaneously calculated using the generalized 3D incompressible Navier-Stokes equation for fluid flow in porous

\footnotetext{
3 www.cgal.org.

4 www.sofa-framework.org.
}

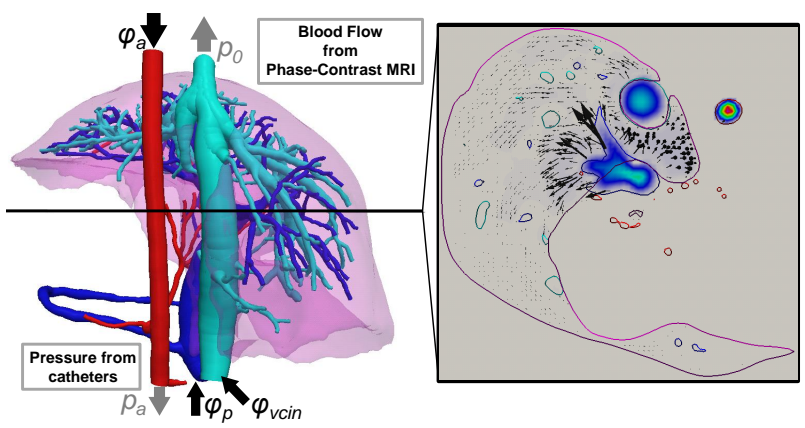

Fig. 9 (Left) Model of the hepatic circulatory system. Black (resp. grey) arrows denote blood flow (resp. outlet pressures) which are fixed as boundary conditions. (Right) Slice showing the computed blood flow in the vessels and parenchyma. The velocity magnitude is color-coded and the velocity vectors are shown in black in the parenchyma.

media [25]. More precisely, writing $\mathbf{v}$ as the blood velocity and $p$ the pressure inside the liver, we solve:

$\frac{\partial \mathbf{v}}{\partial t}+\mathbf{v} \cdot \nabla \mathbf{v}=-\frac{1}{\rho_{b}} \nabla p+\frac{\mu}{\rho_{b}} \nabla^{2} \cdot \mathbf{v} \underbrace{-\frac{\mu(1-\epsilon)^{2}}{\alpha^{2} \epsilon^{2}} \mathbf{v}}_{\mathbf{F}}$

The last term of Eq. 1 is the added force $\mathbf{F}$ that models the total body force due to the presence of a porous medium [26]. F depends on the porosity $\epsilon$ (fraction of blood volume over the total volume) defined through a porosity map (Fig. 8) as described in the previous section. $\rho_{b}$ is the blood density, $\mu$, the shear viscosity of the fluid and $\alpha^{2}$ an effective parameter.

Boundary Conditions. At the border of the liver, no flux boundary conditions are used (Neumann) whereas Dirichlet boundary conditions are applied at the inlets and at the outlet of the large vessels: the portal vein, vena cava and arterial inflows, $\varphi_{p}, \varphi_{v c_{i n}}$ and $\varphi_{a}$ are given from pre-operative Phase-Contrast MR images whereas the vena cava and arterial outlet pressures $p_{0}$ and $p_{a}$ are given from catheterization. Fig. 9 illustrates boundary conditions on a subject-specific geometry.

This method makes the boundary conditions simple to treat: no boundary conditions are fixed on the extremities of the vessels inside the parenchyma thanks to the use of the porosity map, contrary to [9].

\section{Numerical Computation using LBM.}

Eq. 1 is solved using the Lattice Boltzmann Method (LBM) for fast computation on general purpose graphics processing units (GPU). LBM has been developed for CFD and is now a well-established discretization method [27]. In this paper, LBM is used to compute the porous and blood flow circulation in the liver. To this end, an isotropic Cartesian grid with 19-connectivity topology is used (D3Q19 scheme defined in Fig. 10, left) as well as a Multiple-Relaxation-Time (MRT) model, 

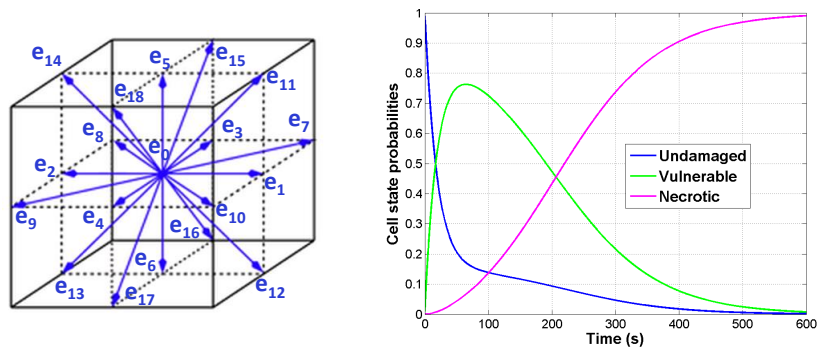

Fig. 10 (Left) D3Q19 scheme used in the LBM computation of the blood flow. (Right) Cell state evolution over time when tissue is heated at $105^{\circ} \mathrm{C}$ during 10 minutes.

for increased stability [28]. At position $\mathbf{x}$ for the edge $\mathbf{e}_{i}$ of the grid, the governing equation is:

$\mathbf{f}\left(\mathbf{x}+\mathbf{e}_{\mathbf{i}} \Delta x, t+\Delta t\right)=\mathbf{f}(\mathbf{x}, t)+\mathbf{A}\left[\mathbf{f}^{e q}(\mathbf{x}, t)-\mathbf{f}(\mathbf{x}, t)\right]+\Delta t \mathbf{g}(\mathbf{x}, t)$

$f_{i}^{e q}(\mathbf{x}, t)=\omega_{i} \rho_{b}\left[1+\frac{\mathbf{e}_{\mathbf{i}} \cdot \mathbf{v}}{c c_{s}^{2}}\right] \quad g_{i}(\mathbf{x}, t)=\omega_{i} \rho_{b} \frac{\mathbf{e}_{\mathbf{i}} \cdot \mathbf{F}}{c_{s}^{2}}$

In this equation, $\mathbf{f}(\mathbf{x})=\left\{f_{i}(\mathbf{x})\right\}_{i=0 . .18}$ is the vector of distribution function with $f_{i}(\mathbf{x})$ being the probability of finding a particle traveling along the edge $\mathbf{e}_{i}$ of the node $\mathbf{x}$ at a given time; $c=\Delta x / \Delta t ; c_{s}^{2}=1 / 3 ; \Delta x$ is the spacing; $\boldsymbol{\omega}=\left\{\omega_{i}\right\}_{i=0 . .18}$ is the vector of weighting factors and $\mathbf{A}$ the MRT matrix. The fluid mass density and velocity are computed from the LBM distributions as $\rho_{b}=\sum_{i=0}^{18} f_{i}(\mathbf{x}, t)$ and $\rho_{b} \mathbf{v}=\sum_{i=0}^{18} \mathbf{e}_{\mathbf{i}} f_{i}(\mathbf{x}, t)+\rho_{b} \mathbf{F} \Delta t / 2$ and are updated at every node of the grid for every time step $\Delta t$.

\subsection{Heat Transfer And Cellular Necrosis Models}

Heat Transfer Model. The coupled bio-heat equation describes how the heat flows from the probe through the liver while taking into account the cooling effect of the blood flow [29]. It depends on the patient-specific anatomy (the segmentation process was presented in Sec. 2.3) and on the blood flow inside the main vessels and the parenchyma considered as a porous medium.

It has be shown [29] analytically and computationally but in $1 \mathrm{D}$ that the coupled bio-heat equations can be simplified into the Pennes model [14] in the case of large vessels, and into the WK model in the case of small vessels. Therefore, the temperature $T$ is computed by solving the following equations, either a reactiondiffusion or an advection-diffusion equation:

$\rho_{t} c_{t} \frac{\partial T}{\partial t}=Q+\nabla \cdot\left(d_{t} \nabla T\right)\left\{\begin{array}{l}+R\left(T_{b 0}-T\right) \\ -\alpha_{v} \rho_{b} c_{b} \mathbf{v} \cdot \nabla T\end{array}\right.$ where $\rho, c, d$ are the density, heat capacity, conductivity; subscripts $t$ and $b$ stand for tissue and blood respectively. $Q$ is the source term, $\alpha_{v}$, the advection coefficient, $R$, the reaction coefficient (it corresponds to $H /(1-\epsilon)$ from [8] ) and $T_{b 0}$ the blood temperature (assumed constant) in large vessels. In this case, the advection is the transport mechanism of the heat by the blood due to its motion, whereas the diffusion is the transport mechanism due to a temperature difference within a same region but without any motion. The Pennes Model is solved in the large visible vessels and the WK model in the liver parenchyma. Our model includes the heat sink effect of all hepatic vessels (veins and arteries) as well as the effect of the blood flow within the parenchyma considered as a porous medium.

A weak coupling is considered: the blood flow has an influence on the temperature distribution through the advection term in the WK model and through the reaction term in the Pennes model but the temperature does not affect the blood flow (coagulation is not considered here), which allows us to speed up the calculations since the blood flow distribution is computed only once, at the beginning of the simulation, and is run until a steady state is reached.

Cellular Necrosis Model. A three-state model [30] is coupled with the bioheat equation to compute tissue necrosis. Each cell has a probability to be either undamaged (U), vulnerable (V) or necrotic (N). Those probabilities vary with the temperature spatially and temporally according to the following transition diagram:

$$
[\mathrm{U}] \stackrel{\beta(T)}{\overleftarrow{\gamma}}[\mathrm{V}] \stackrel{\delta(T)}{\longrightarrow}[\mathrm{N}]
$$

where $\beta(T)=\bar{\beta} e^{T / T_{k}}, \delta(T)=\alpha \beta(T)$ and $\gamma$ are the transition rates. Unlike in [16], a constant $\alpha$ is introduced $(\alpha=\bar{\delta} / \bar{\beta}(1+10 N))$ to decouple the damage rate from the vulnerable rate coefficient, so that three distinct transition rates are considered to allow cells to reach the vulnerable state. This diagram results in three coupled ODEs (Eq. 3), solved with a first order explicit scheme on the same grid and with the same time step as the bioheat equation.

$\left\{\begin{array}{l}\frac{d U}{d t}=-\beta(T) U+\gamma V \\ \frac{d V}{d t}=\beta(T) U-(\gamma+\delta(T)) V \\ \frac{d N}{d t}=\delta(T) V\end{array}\right.$

The property: $U+V+N=1$ is also imposed at each point. (Fig. 10, right) represents the solution of Eq. 3 
at one vertex over time if a constant temperature of $105^{\circ} \mathrm{C}$ is applied.

For both heat transfer and cell death models, parameters are initially set to values from the literature [8] reported in Table. 1, assumed similar for surrogate tumor and hepatic tissue.

The cell death model is strongly coupled to the bioheat equation as the heat capacity depends on the state of the cell $\left(c_{t}^{U}, c_{t}^{V}\right.$ and $c_{t}^{N}$ correspond respectively to the heat capacity of undamaged, vulnerable and necrotic tissue) and the conductivity $d_{t}$ depends on the temperature through $d_{t}=\bar{d}_{t} *\left(1+1.61 *(T-310) \cdot 10^{-3}\right)$ as in [8]. All the remaining parameters are constant.

Numerical Computation using LBM. Eq. 2 are also solved using LBM with a MRT model. In RFA, it has been used to compute heat transfer and validated through a comparison with an analytical solution [9], for a similar accuracy as FEM, though it has the advantage to be easily parallelized in GPU.

An isotropic Cartesian grid with 7-connectivity topology has been found sufficient [9]. Neumann conditions at the liver boundary defined as a level set function are applied. For a time step of $\Delta t=0.5 \mathrm{~s}$ and a spatial resolution of $\Delta x=0.5 \mathrm{~mm}$, better than real-time computation can be achieved on a desktop machine (Windows 7, Intel Xeon, 3.30GHz, 16GB RAM, 12 CPUs, Nvidia Quadro K5000 4.0 GB). For example, it took around 10 mins to compute 17 mins of ablation of Pig 4-1.

Table 1 Nominal parameter values from the literature [8].

\begin{tabular}{|c|c|c|}
\hline Notation & Parameter Name & Nominal \\
\hline$T_{k}\left({ }^{\circ} \mathrm{C}\right)$ & $\begin{array}{l}\text { parameter of cell death } \\
\text { model }\end{array}$ & 40.5 \\
\hline $\bar{\beta}\left(\mathrm{s}^{-1}\right)$ & damage rate coefficient & $3.3 \times 10^{-3}$ \\
\hline$\gamma\left(\mathrm{s}^{-1}\right)$ & recovery rate coefficient & $7.7 \times 10^{-3}$ \\
\hline $\bar{\delta}\left(\mathrm{s}^{-1}\right)$ & vulnerable rate coefficient & $3.3 \times 10^{-3}$ \\
\hline$c_{t}^{V}\left(\mathrm{~J}(\mathrm{~kg} \mathrm{~K})^{-1}\right)$ & $\begin{array}{l}\text { heat capacity of vulnerable } \\
\text { tissue }\end{array}$ & $3.6 \times 10^{3}$ \\
\hline$c_{t}^{N}\left(\mathrm{~J}(\mathrm{~kg} \mathrm{~K})^{-1}\right)$ & $\begin{array}{l}\text { heat capacity of necrotic tis- } \\
\text { sue }\end{array}$ & $0.67 \times 10^{3}$ \\
\hline$c_{t}^{U}\left(\mathrm{~J}(\mathrm{~kg} \mathrm{~K})^{-1}\right)$ & $\begin{array}{l}\text { heat capacity of undamaged } \\
\text { tissue }\end{array}$ & $3.6 \times 10^{3}$ \\
\hline $\bar{d}_{t}\left(\mathrm{~W}(\mathrm{~m} \mathrm{~K})^{-1}\right)$ & heat conductivity & 0.512 \\
\hline $\mathrm{R}\left(W\left(m^{3} K\right)^{-1}\right)$ & reaction coefficient & $27.1 \times 10^{4}$ \\
\hline$\alpha_{v}$ & advection coefficient & 0.11 \\
\hline
\end{tabular}

\subsection{Heating Power and Cooling Temperature Computation}

We assume that the equilibrium between probe and tissue temperature is reached. During the heating period, a Dirichlet boundary condition is used to fix the temperature at the point sources of the probe tips: RFA is simulated by imposing as input the temperatures measured at the five thermistors in a small neighborhood around the probe tips: a layer of several voxels. Thus simulating the source term $Q$ from Eq. 2. The four remaining tip temperatures are linearly interpolated from these values. Imposing temperature values does not prevent of observing large temperature gradient next to the tips. This large gradient of temperature is in fact observed in our simulations. The heating stops at time $t=t_{a}$. During this period $\left(t<t_{a}\right)$ the heating power can be computed, whereas the cooling temperature can be computed when $\left(t>t_{a}\right)$.

Heating Stage. We assume that the measured power is strongly correlated (proportional) to the heat power $P(t)$ delivered through radio-frequency to heat the liver tissue. Proportionality is assumed to account for power dissipation due to electrical resistance, and the unknown surface ratio of the probe being heated, but also since we do not model the heat loss by Joule effect. The heat power $P(t)$ delivered to the tissue can be computed at each time step of the simulation from the bioheat equation according to Fourier's law:

$P(t)=\alpha \int_{S} d_{t} \frac{\partial T(t)}{\partial \mathbf{n}} d S$

$S$ is the probe surface, $\mathbf{n}$ is the outer normal at that surface and $\alpha$, the proportionality coefficient $(\alpha=0.4469)$, found by matching the peak value of the measured power for Pig 1-1 with the peak value of the simulated power with personalized parameters. This value is then used in all the computations.

Cooling Stage. In the absence of any delivered heat power, the nine tips of the probe cool down at a speed which depends on the local conductivity $d_{t}$ and the heat capacity $c_{t}$. Thus, during the cooling period $t_{c}-t_{a}$ (cooling stops at time $t=t_{c}$ ), the cooling temperature can be simulated.

\subsection{Parameter Estimation from Probe Measurements}

During the intervention, the delivered power and the temperature distribution are measured by the ablation probe itself. We explore how these information can be used to estimate apparent values of some model parameters. During the heating phase, the simulated heat power $P_{s}$ can be compared to the measured one $P_{m}$. During the cooling phase, the simulated temperatures $\mathbf{T}_{\mathbf{s}}$ can be compared with the measurements $\mathbf{T}_{\mathbf{m}}$ read from five tips of the probe (four tips do not have any thermistors).

In this study, the nominal parameters in the heat transfer and cellular necrosis equation come from the 
literature. After a non reported sensitivity analysis, we choose to estimate apparent values of the heat capacity $c_{t}^{U}$ and the constant part of the conductivity $\bar{d}_{t}$ as they mainly influence the delivered power, the temperature distribution and the size of ablated regions. As temperature maps are not readily available, these two apparent values of the parameters are obtained by minimizing the following cost function:

$$
\begin{aligned}
& \arg \min _{c_{t}^{U}, \bar{d}_{t}}\left(\frac{1}{t_{a}} \sum_{t=0}^{t=t_{a}-\Delta t_{m}}\right. \frac{\left(P_{m}(t)-P_{s}(t)\right)^{2}}{\sigma_{P_{m}}^{2}}+ \\
&\left.\frac{1}{t_{c}-t_{a}} \sum_{t=t_{a}}^{t_{c}} \frac{\left(m_{T_{m}}(t)-m_{T_{s}}(t)\right)^{2}}{\sigma_{T_{m}}^{2}}\right)
\end{aligned}
$$

$\Delta t_{m}$ is the measurement time step, $\sigma_{P m}$ and $\sigma_{T m}$ are the standard deviations associated with the heat power and the temperature, both of them evaluated from the variability in the available observations and equal to 13.3 $\mathrm{W}$ and $5.1^{\circ} \mathrm{C}$ in our experiments. $\sigma_{P m}$ acts as a normalization factor in Eq. 5. It is computed from the twelve ablations at one time point during the heating phase $(t=50 \mathrm{~s})$, time at which all power curves are coherent (Fig. 11).

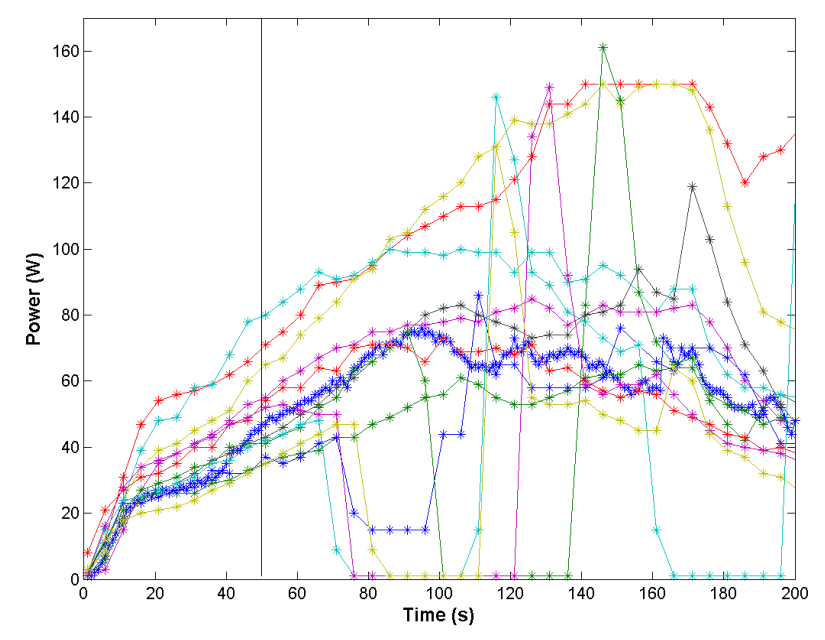

Fig. 11 Plot of the 12 delivered power curves for the first 200 seconds. The mean and standard deviation are computed at $t=50 \mathrm{~s}$, This time is a good compromise since it is not too early, avoiding the boundary condition effect, and not too late, before the cell death could affect the delivered power. The wild fluctuations are due to the different heating/cooling phases.

$\sigma_{T m}$ is computed from the 5 temperature curves extracted from the cooling phase of one ablation (Pig 11 ), where all the temperature curves are coherent. We did not use all the twelve ablations as their cooling stage did not start at the same time. It is defined as follow:
$\sigma_{T m}^{2}=\frac{1}{t_{c}} \sum_{t=t_{a}}^{t=t_{c}}\left(\frac{1}{5} \sum_{i=1}^{5}\left(T_{i}(t)-m_{T}(t)\right)^{2}\right) \quad m_{T}(t)=\frac{1}{5} \sum_{i=1}^{5} T_{i}(t)$

To cope with the uncertainty in the rotation of the probe along its axis, the mean tip temperature $m_{T}(t)$ is used for the personalization instead of directly mapping the tip temperatures.

\section{Results}

\subsection{Evaluation of the Pre-processing}

Evaluation of the Registration. The accuracy of the registration is visually checked by an expert (Fig. 12). Whenever available, the non-rigid transformation is applied to the post-operative vessel and/or tumor meshes and they are compared to their pre-operative equivalent meshes. Point-to-mesh errors from pre- to registered post-operative meshes are computed for Pig 2 (Table 2). Before the non-rigid registration, all the meshes are centered. The error is decreased after the registration except for tumor 1 where the two meshes are already in good agreement before the registration.

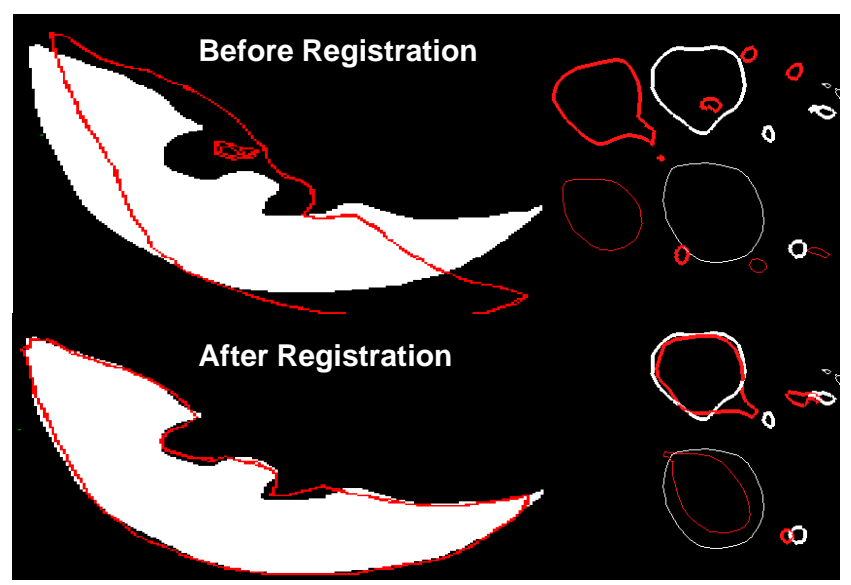

Fig. 12 (Left) Superposition of the post-operative mesh model in red on the pre-operative binary image; (Right) Comparison between the registered post-operative vessel meshes in red (portal vein in thick, vena cava in thin lines) and the pre-operative ones.

Evaluation of the Smooth Vessels Trees. In order to evaluate the creation of the smooth vessels trees, DICE, Positive Predictive Value (PPV) and sensitivity scores between the original and the smoothed vessel images are computed for Pig 4 (Table 3). They show a good correlation between the original segmented vessels and the generated ones. 
Table 2 Point-to-mesh errors from the pre- to the postoperative meshes in millimeter before and after the registration for Pig 2. Before the registration, all the meshes are centered.

\begin{tabular}{lll}
\hline Mesh & Before registration & After registration \\
\hline hepatic veins & $4.53 \pm 2.13$ & $2.30 \pm 0.97$ \\
portal vein & $6.13 \pm 3.81$ & $4.88 \pm 4.38$ \\
arteries & $21.34 \pm 23.25$ & $17.60 \pm 19.71$ \\
tumor 1 & $2.25 \pm 1.38$ & $2.80 \pm 1.49$ \\
tumor 2 & $8.80 \pm 6.51$ & $5.28 \pm 4.50$ \\
liver & $6.91 \pm 3.57$ & $3.53 \pm 1.60$ \\
\hline
\end{tabular}

Table 3 Dice, Positive Predictive Value (PPV) and sensitivity between the original and the smoothed vessel images for Pig 4.

\begin{tabular}{llll}
\hline Mesh & DICE (\%) & PPV (\%) & Sensitivity (\%) \\
\hline arteries & 93.1 & 93.9 & 92.3 \\
portal vein & 91.3 & 85.3 & 98.3 \\
hepatic veins & 84.9 & 93.8 & 77.5 \\
\hline
\end{tabular}

Verification of the CFD Solver on a Synthetic Case. A cylindrical mesh with spherical inlet and outlet (Fig. 13), is used to validate the CFD solver in the case of a porous medium. Blood flow is set at the inlet and pressure at the outlet. The flow entering the vessel $\Phi_{i n}$ should entirely go out: $\Phi_{\text {in }}=\Phi_{\text {out }}$, in the case of a single vessel (case 1: Fig. 13, left) but also in the case where the flow crosses a porous medium, a porosity map is used to emulate two veins and the liver parenchyma (case 2: Fig. 13, right). The porosity is set to 1 inside the vessels, 0.04 in the endothelium and 0.1 outside. The mass conservation is verified by comparing the flow through the surface of the "vessel" at $z=20 \mathrm{~mm}$ : $\Phi_{i n}$ and at $z=80 \mathrm{~mm}$ : $\Phi_{\text {out }}$. In both cases, a difference between these two fluxes of $3 \%$ is achieved.

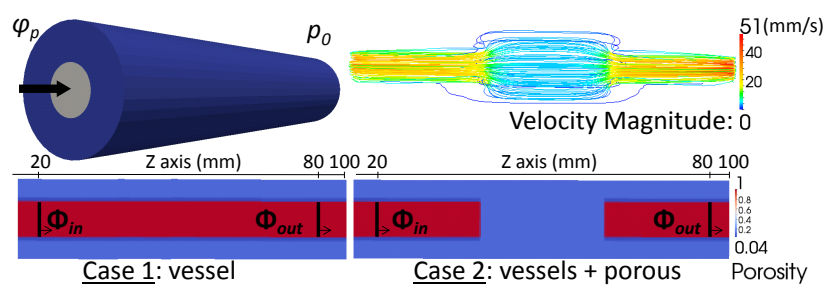

Fig. 13 Synthetic case used to validate the CFD solver. (Top Left) A cylindrical mesh with spherical inlet and outlet is used. The boundary conditions are the inflow and the outlet pressure. (Top Right) The velocity field given by the CFD solver in the second case. (Bottom Row) The porosity field used in the two cases.
4.2 Verification of the Parameter Estimation

Framework

As illustrated on Fig. 1, the framework enables parameter estimation too.

Synthetic Data Generation. In order to verify the parameter estimation framework, we consider a synthetic case on a simplified regular cuboid domain, to speed-up the process (Fig. 14, left). As we try to estimate the heat conductivity and the heat capacity, the advection is not considered here.

We apply the typical clinical RFA protocol: during three minutes, the temperature is increased linearly at the tips of the probe to reach $105^{\circ} \mathrm{C}$, and then maintained for six minutes. Finally a cooling stage is observed (no temperature imposed) for three more minutes. The extent of the necrotic area, delivered power and temperature during the cooling stage are simulated and then used as ground truth to estimate the parameters: $\bar{d}_{t}$ and $c_{t}$ by minimizing the cost function (Eq. 5). Comparison of Optimization Methods. We compare two gradient-free optimization methods available in DAKOTA ${ }^{5}$ : the Constrained Optimization BY Linear Approximations (COBYLA) and the pattern search method (PS). Those methods are sequential trust-region algorithms, therefore they do not rely on initial values, but explore the whole domain of parameters in a first stage. The range of parameters values used [7] are reported in Table 4 and the other parameters are fixed to the nominal values (Table 1 ).

First, a ground truth is considered using the values of $c_{t}$ and $\bar{d}_{t}$ found in Sec. 5 corresponding to healthy swine tissue. Using COBYLA, we estimate the parameters with $0.8 \%$ error on $\bar{d}_{t}$ and $0.6 \%$ on $c_{t}$ in 30 minutes after 22 iterations with a cost function value of $7.810^{-5}$ and a mean of the symmetric point-to-mesh error of $10^{-4} \mathrm{~mm}$. Similarly, using PS, we manage to obtain the estimated parameters with $0.4 \%$ of error on

\footnotetext{
${ }^{5}$ http://dakota.sandia.gov - multilevel framework for sensitivity analysis.
}
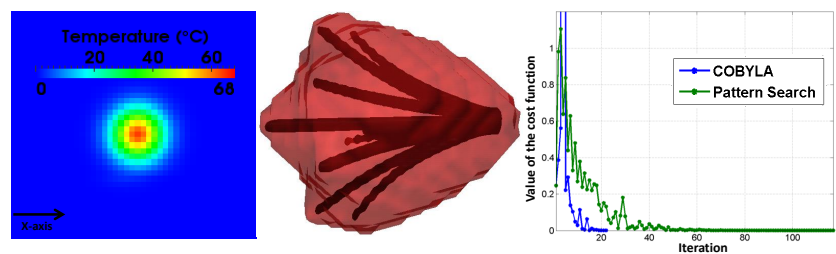

Fig. 14 (Left) Set-up of the synthetic case: the temperature map around one tip of the probe, and the necrotic area (red) created around the probe (dark). (Right) Convergence curves of the COBYLA algorithm (blue) and of the pattern search algorithm (green). The cost function is shown with respect to the number of iterations. 


\begin{tabular}{llrc}
\hline Notation & Parameter Name & Min & Max \\
\hline$c_{t}^{U} \mathrm{~J}(\mathrm{~kg} \mathrm{~K})^{-1}$ & $\begin{array}{l}\text { heat capacity of un- } \\
\text { damaged tissue }\end{array}$ & 18 & 54 \\
$\bar{d}_{t} \mathrm{~W}(\mathrm{~m} \mathrm{~K})^{-1}$ & $\begin{array}{l}\text { heat conductivity } \\
0.256\end{array}$ & 0.768 \\
\hline
\end{tabular}

Table 4 Ranges of parameters values explored in the optimization framework.

$\bar{d}_{t}, 0.3 \%$ on $c_{t}$ in 154 minutes after 117 iterations with a cost function value of $7.210^{-6}$ and a similar mean of the symmetric point-to-mesh error of $10^{-4} \mathrm{~mm}$. The convergence curves of both optimizations are shown on (Fig. 14, right). We choose the COBYLA method to perform the personalization as it requires less iterations of the forward model to estimate the parameters with similar accuracy as PS.

Evaluation. Cirrhotic tissue behaves like a thermal insulator, preventing to heat outside the tumor targeted for the ablation, leading to the so-called "oven effect" [31]. In order to mimic this effect, the heat conductivity is divided by two (to the best of our knowledge, no value are reported in the literature) to generate the ground truth, which differs from the one obtained with the nominal value (Fig. 15, right). We manage to find the correct cirrhotic value of $\bar{d}_{t}$ (Fig. 15, left).

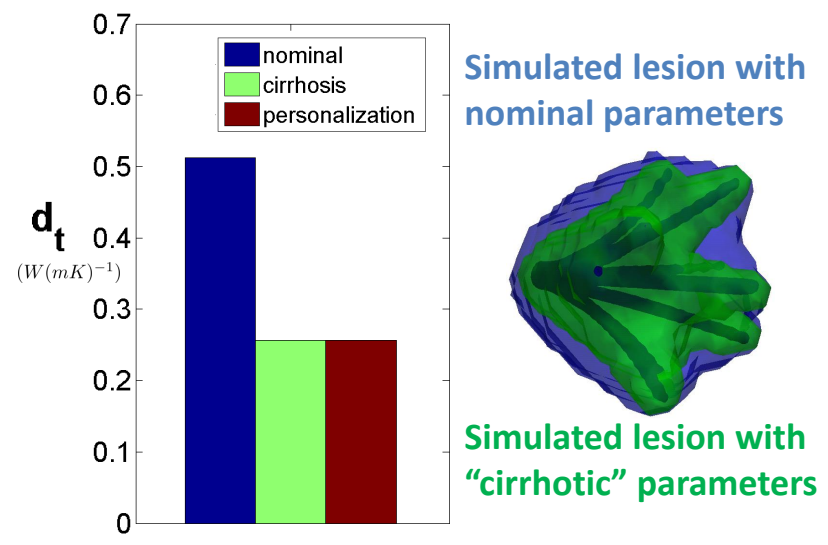

Fig. 15 (Left) The parameter estimation framework leads to the correct "cirrhotic" value of $\bar{d}_{t}$. (Right) The lesion obtained with the "cirrhotic" $\bar{d}_{t}$ in green is enclosed within the lesion obtained with the nominal value in blue.

We verified on a synthetic case, that our method allows to find "cirrhotic" value of the heat conductivity in the case of a diseased liver in the absence of noise and model error.

\subsection{Evaluation on Swine Data}

We now evaluate our method on the available swine data, whose liver tissues are healthy.

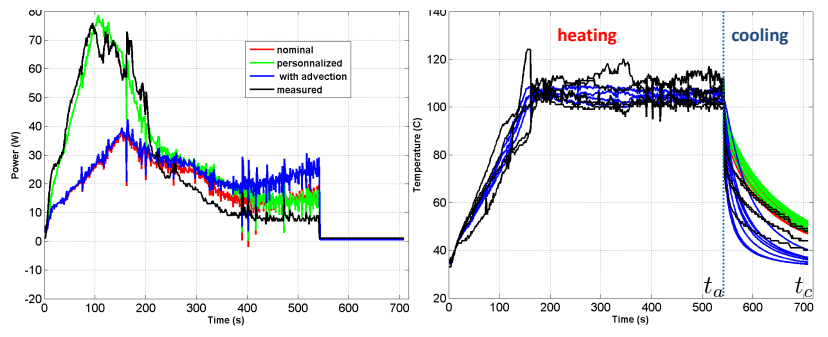

Fig. 16 Temperature and delivered power evolution for Pig 11. 3 different computations: with nominal parameters (green curves), personalized parameters (red curves), and with advection (blue curves) are compared with actual measurements (black curves). (Left) The error on the power evolution computed over the heating phase is reduced from 19.9 to $7.5 \mathrm{~W}$ after personalization and stays constant with advection (20.8 W). (Right) The error on the temperature evolution computed over the cooling phase is reduced from $10.3^{\circ} \mathrm{C}$ to $9.7^{\circ} \mathrm{C}$ with the advection, but stays constant after personalization (11.1 $\left.{ }^{\circ} \mathrm{C}\right)$. The advection clearly affects the temperature evolution.

During our experiments, the measured electrical power appears consistently to reach its maximum during the increase in temperature, before the plateau of $105^{\circ} \mathrm{C}$ (Fig. 16, left) in all cases. Given the considered model and the Fourier's law used to compute the delivered power (Eq. 4), this phenomena cannot be explained by a constant heat capacity which would lead to a peak after the plateau is reached. Instead, following a non reported sensivity analysis, we propose to adapt the necrosis model of [16], so that the cells reach their vulnerable state faster, which is one way to explain this observation. By modifying $\bar{\delta}$, the two forward transitions $[U] \rightarrow[V]$ and $[V] \rightarrow[N]$ are decoupled so that tissues can reach very quickly their vulnerable state which entails a significant change of $c_{t}$ (Table 5).

The model is evaluated on twelve ablations performed on five swines (some swines have several surrogate tumors). While the large and CT-visible hepatic vessels have a cooling effect affecting the shape of the thermal lesion [13], the effect of the hepatic perfusion due to the small vessels non visible on CT images has not been clearly identified so far. For this reason, the simulations are performed without the advection term, capturing the hepatic perfusion, except in Sec. 4.3.2 in order to understand its effect on the necrotic lesion. By keeping the reaction term, we only consider the heat sink effect of the blood on the large visible vessels in each simulation. The applied RFA protocol is not exactly the same for all ablations. Eight ablations are performed through several short cooling and heating periods, whereas the other four ablations included only one long final cooling stage after a continuous heating period. For all pigs, nominal values of parameters (Table 1) are employed. In each case, the simulated lesion 
is compared to the ground-truth. Fig. 17 shows results for Pig 4-2.

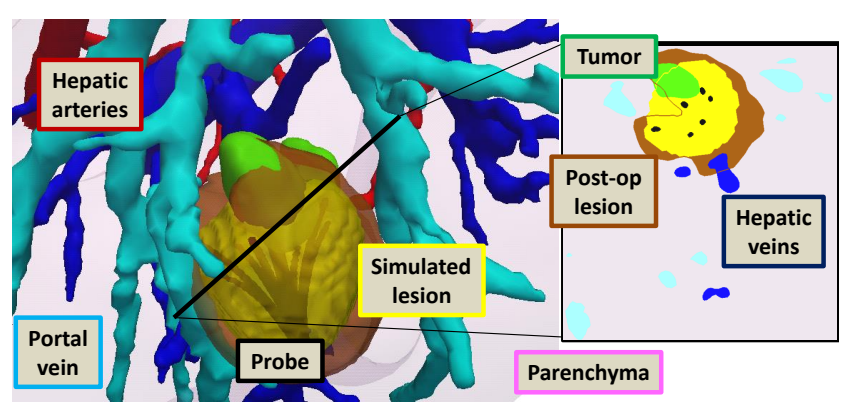

Fig. 17 (Left) The simulated lesion in yellow is showed around the RFA probe in the subject-specific geometry on Pig 4-2. (Right) A single orthogonal slice shows that the simulated lesion is qualitatively close to the registered postoperative lesion in brown: point-to-mesh error of $4.6 \pm$ $3.9 \mathrm{~mm}$. The color of the boxes correspond to the color of the respective regions.

Quantitatively, symmetric point-to-mesh errors implemented like in [32] computed between the simulated lesion and the registered post-operative ground-truth show suitable prediction of the necrotic extent (in red on Fig. 18): average over all ablations of $5.3 \pm 3.6 \mathrm{~mm}$ of mean point-to-mesh error, smaller than the targeted surrogate lesions diameter (around 2 to $3 \mathrm{~cm}$ ), which can be considered as sufficient for clinical applications. Similarity scores averaged over all ablations are as follows: DICE: $44 \%$, sensitivity: $47 \%$, and PPV: $53 \%$ (in red on Fig. 19). We achieve errors of $26 \mathrm{~W}$ and 5.1 ${ }^{\circ} \mathrm{C}$ on average between measured and simulated values of cooling temperature and delivered power (in red on Fig. 20), which is good compared to the targeted ablation temperature of $105{ }^{\circ} \mathrm{C}$ and the maximal power of $150 \mathrm{~W}$.

\subsubsection{Parameter Personalization}

COBYLA is used to minimize the cost function (Eq. 5) as only a few forward simulations (typically 20) are required. As the data originates from healthy pigs of similar age and weight, we hypothesize that their parameters would be similar too. The vulnerable rate coefficient is also adjusted to match the raise in delivered power as detailed in Sec. 4.3. The minimization of the error between measured and simulated values of power and temperature is done only on two tumors with a long final cooling stage (2 different Pigs: Pig 1-1 and Pig 4-3) as it is long enough to observe reliably the effect of the conductivity $\bar{d}_{t}$, yielding two sets of personalized values. The values independently found on the two swines are really close: the same value of heat capacity $c_{t}^{U}$ is

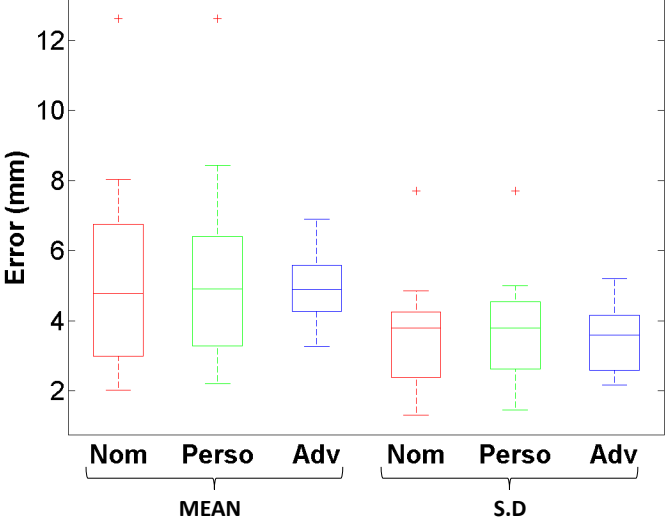

Fig. 18 Results for three different computations: with nominal parameters, with personalized parameters and with the advection term. For the computation with the advection term, only 6 ablations are considered. Mean and standard deviation of the point-to-mesh in mm computed on the surface of the lesion.

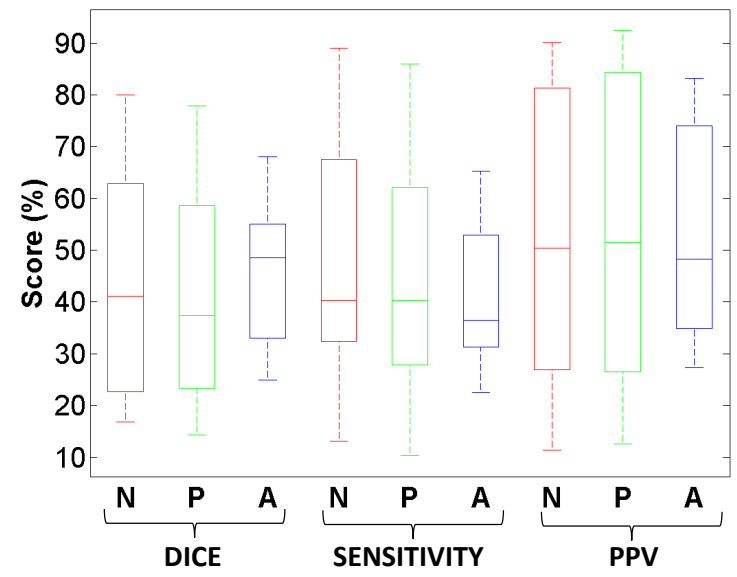

Fig. 19 Similarity scores (DICE, Sensitivity and Positive Predictive Value) in \% computed on the volume of the lesion.

estimated, and the conductivity values are almost equal to the nominal value as expected (Table 5). The estimated parameters are then used on the ten remaining cases to evaluate the discrepancy in terms of temperature, delivered power and necrotic area. Small errors were obtained in those cases too, without previously having fit the parameters for those tumors. On average, the temperature and power errors are $7.8^{\circ} \mathrm{C}$ and $20.4 \mathrm{~W}$ (in green on Fig. 20), the mean of the point-tomesh error is $6.0 \mathrm{~mm}$ (in green on Fig. 18) and similarity scores are DICE: $42 \%$, sensitivity: $43 \%$, PPV: $55 \%$ as illustrated in green on Fig. 19. Qualitatively, as one can see on Fig. 21 (green curves), the simulated heat power and temperature are close to the heat power and the temperature given by the RFA probe itself. On average, the error is reduced after the use of personalized parameters: from $35 \mathrm{~W}$ to $30 \mathrm{~W}$ and from $5.9{ }^{\circ} \mathrm{C}$ to 


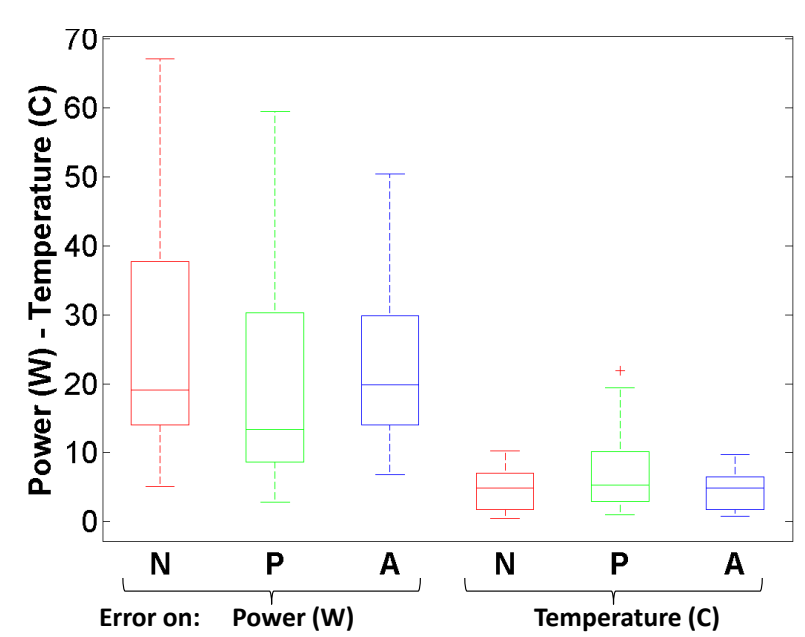

Fig. 20 (Left): Error computed during the heating phase on the power in W (Right): Error computed during the cooling phase on the temperature in ${ }^{\circ} \mathrm{C}$.

$1.9{ }^{\circ} \mathrm{C}$. It is mainly reduced on the first heating period (from $50 \mathrm{~W}$ to $27 \mathrm{~W}$ ). During the cooling phase, the simulated (non-imposed) temperature is compared to the measured one, whenever measurement is available (the RFA power was turned off during some cooling phases).

Table 5 Comparisons between personalized and nominal values of estimated parameters.

\begin{tabular}{lllll}
\hline \multicolumn{2}{c}{ Notation Nominal } & \multicolumn{2}{c}{ Personalized Values } & Automatic \\
& Value & on Pig 1-1 & on Pig 4-3 & optimisation \\
\hline$\delta$ & $3.3 \times 10^{-3}$ & $1 \times 10^{-4}$ & $1 \times 10^{-4}$ & No \\
$c_{t}^{U}$ & $3.6 \times 10^{3}$ & $3.6 \times 10^{1}$ & $3.6 \times 10^{1}$ & Yes \\
$\bar{d}_{t}$ & 0.512 & 0.614 & 0.512 & Yes \\
\hline
\end{tabular}

\subsubsection{Effect of the Advection}

To quantify the effect of hepatic perfusion, the simulations are performed by adding the advection term for all pigs whenever it is possible to segment the vena cava inlet (not possible for Pig 2 and 4). On average, the effect of the advection cannot be clearly highlighted. The mean of the point-to-mesh error over the 6 ablations computed with advection is $5.0 \pm 3.5 \mathrm{~mm}$ (in blue on Fig. 18), the average similarity scores are: Dice: $46 \%$, sensitivity: $42 \%$ and PPV: $53 \%$ (in blue on Fig. 19). On average, the error on the simulated power is $22 \mathrm{~W}$ and $4.7{ }^{\circ} \mathrm{C}$ on the temperature (in blue on Fig. 20). Those values are comparable to the values obtained without the advection term. As one can see on Fig. 21 (blue curves), the errors remain constant with the advection term $\left(35 \mathrm{~W}\right.$ and $\left.6.3{ }^{\circ} \mathrm{C}\right)$. In some cases, the advection reduces the error in term of necrotic extent and tem-

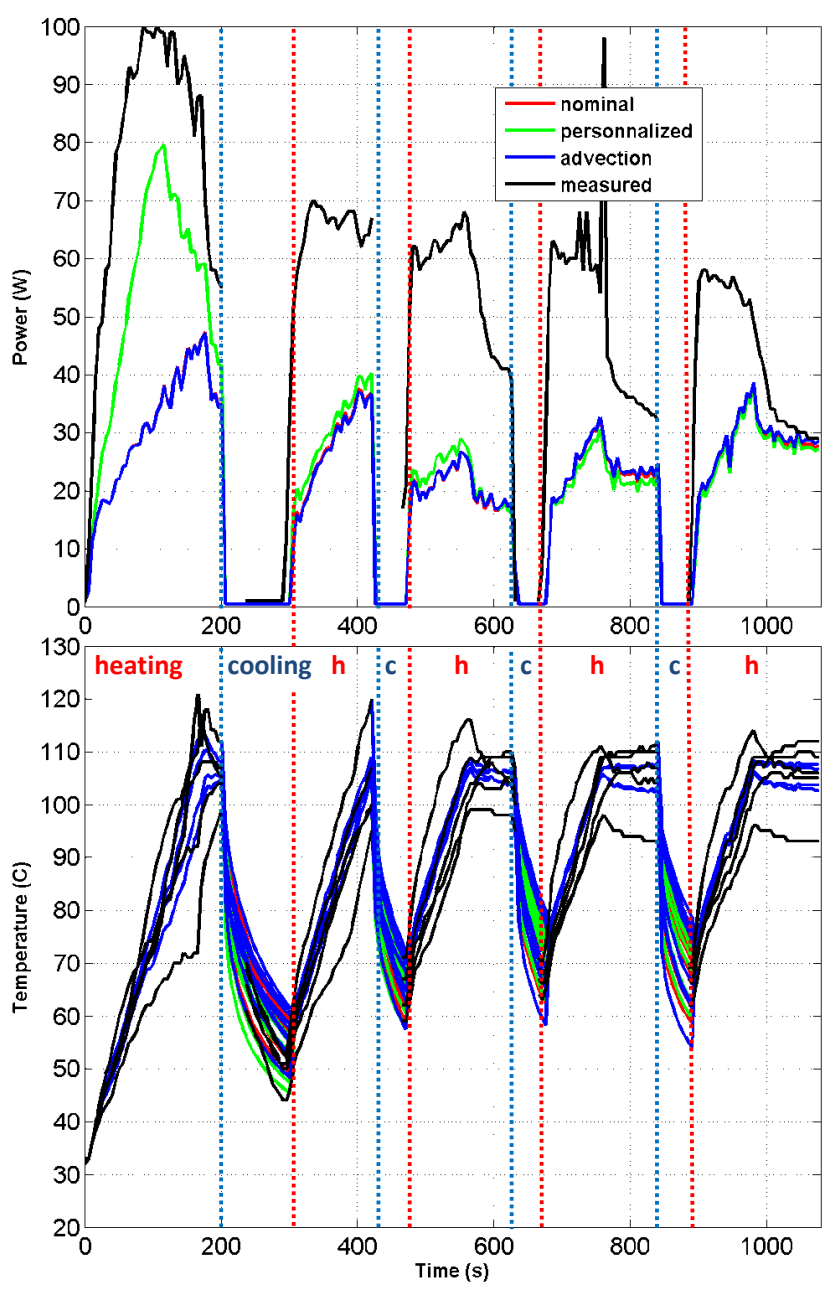

Fig. 21 Temperature and delivered power evolution for Pig 31. 3 different computations: with nominal parameters (green curves), personalized parameters (red curves), and with advection (blue curves) are compared with actual measurements (black curves). During the heating phase, the temperature is imposed: 5 temperatures are directly imposed from the 5 thermistors measurements (superposition of blue and black curves) and the 4 remaining temperatures are imposed from a linear interpolation from those measurements ( 4 blue curves). The red curves are really similar to the blue curves, and they can hardly be distinguished.

perature distribution (Pig 5-1), but not always (Pig 1$1)$. The advection term always change the shape of the necrotic extent (Fig. 22).

\subsubsection{Effect of the Registration}

One important part of the validation is the comparison of the simulated necrotic extent with a lesion segmented on a post-operative image registered to the preoperative image. The modality of the post-operative image is not always the same (CT or MR). The resolution and the size of the post-operative image do not always allow an accurate registration of the lesion, as for Pig 2- 


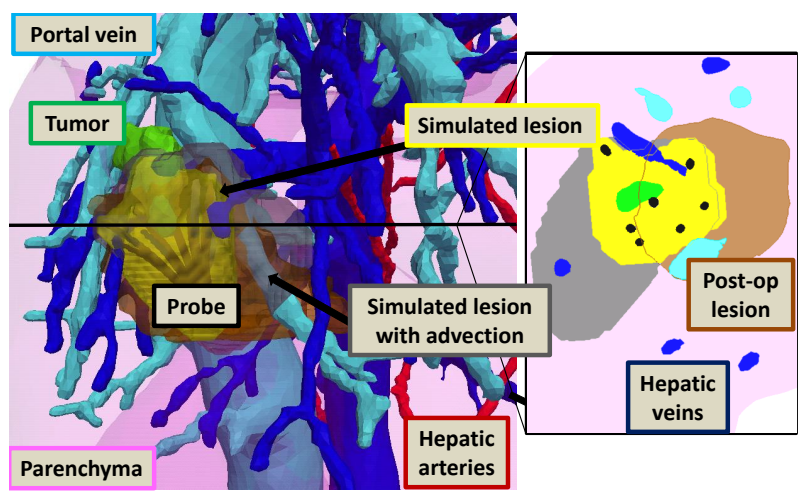

Fig. 22 Comparison between the simulated and the postoperative necrotic areas on Pig 1-1. (Left) The post-op lesion is showed around the RFA probe in the subject-specific geometry. (Right) Single slice showing the simulated lesions with (grey) and without (yellow) advection compared to the registered post-operative lesion (brown). The advection has an effect on the shape of the lesion, however in this case, it does not improve the point-to-mesh error: $6.9 \pm 5.2 \mathrm{~mm}$ vs $4.8 \pm$ $4.4 \mathrm{~mm}$. The box colors correspond to the color of the regions.

2 (resolution: $0.78 \times 0.78 \times 4.8 \mathrm{~mm}$, size: $384 \times 384 \times 36 \mathrm{~mm}$ ). To check the sensitivity of the computation to the registration, a new algorithm is used for this tumor only. We rigidly translate the post-operative lesion so that the barycenter of the post-operative lesion coincides with the barycenter of the simulated necrotic extent (Fig. 23, right). With this simple registration method, the results are significantly improved. The lesion registration strongly affects the error measured between the computed necrotic area and the ground truth.

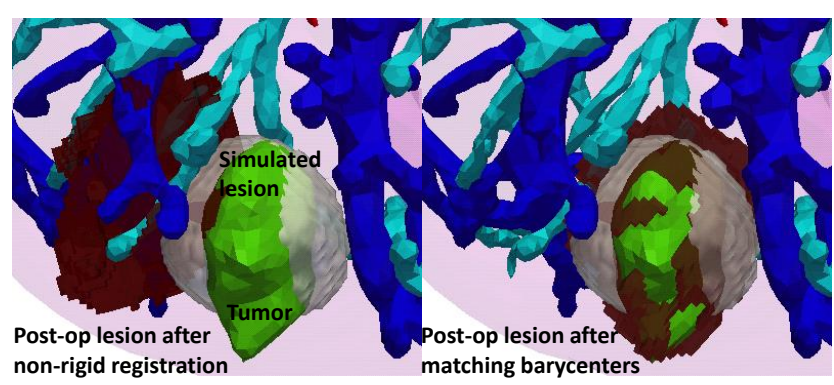

Fig. 23 Effect of the registration on Pig 2 (Left) The postoperative lesion after the non-rigid registration is showed in the subject-specific geometry. The point-to-mesh error is 8.4 $\pm 5.0 \mathrm{~mm}$ (Right) The post-op lesion after matching barycenters. The point-to-mesh error reduces to $3.6 \pm 2.9 \mathrm{~mm}$.

\subsubsection{Effect of the Probe Position}

The position of the probe is known from an intra-operative MRI. In some cases, the probe artifact and the image contrast do not allow an accurate registration, as for
Pig 3-2. A new computation is performed for this tumor to check the sensitivity of the computation to the probe position. The same configuration is used except that we manually put the probe inside the registered post-operative lesion (position 2 in Fig. 24).

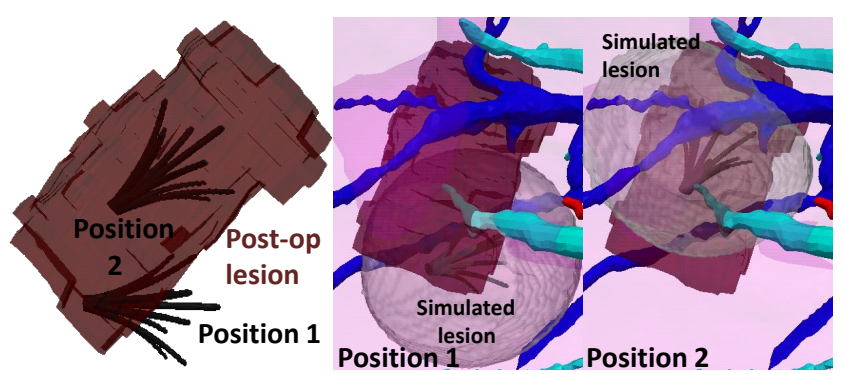

Fig. 24 (Left) The post-operative lesion is showed around 2 possible positions for the RFA probe on Pig 3-2. (Right) Zoom on the ablation area, the simulated lesion is closer to the registered post-operative lesion when the probe is in position 2, point-to-mesh error of $6.2 \pm 4.0 \mathrm{~mm}$ versus $12.6 \pm 7.7 \mathrm{~mm}$ in position 1 .

With this probe position, the results are significantly improved. The probe position affects the error measured between the computed necrotic area and the ground truth (Fig. 24). It is equivalent to a registration error since by moving the probe, we move the computed necrosis.

\section{Discussion}

We presented a first quantitative evaluation of RFA computation, which combines a multi-physics model and multi-modal medical images from a pre-clinical study. Despite possible biases in the probe location and in establishing correspondences from the post- to the preoperative images due to registration errors, our model provided new insights.

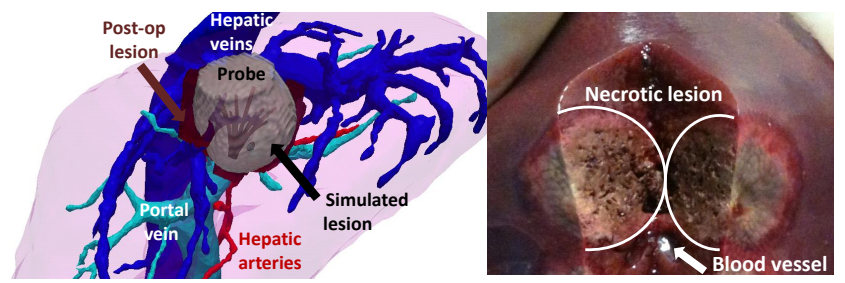

Fig. 25 (Left) The simulated necrotic extent around the RFA probe, close to the border of the liver from Pig 3-3. The postoperative lesion is shown in brown. (Right) The liver extraction confirms that the necrotic lesion is subcapsular. 


\subsection{Model Limitations}

The proposed model has several limitations. We assume that tumor and healthy hepatic tissue have similar properties. Experiments could be performed to assess the effect of different spatial properties on the resulting necrosis. The measured power is assumed to be proportional to the power delivered through radio-frequency. The Neumann boundary condition (no heat transfer through the liver surface) may not be valid. If the ablation location is subcaspsular (Pig 5-1, 4-1, 3-3), the volume of the simulated ablation becomes larger, as the heat bounces back on the border and increases artificially the heat diffusion inside the liver (Fig. 25). However, the liver has a high regulation capacity thanks to its rich vascular system. If the heat is applied close to the liver border, it is not clear how it dissipates, whether or not it will affect the neighboring organs. Moreover, the weak coupling between the heat transfer model and the CFD model does not allow to consider coagulation or the cessation of perfusion at temperature above $60{ }^{\circ} \mathrm{C}$, which is known to have a major impact on ablation lesion size. However this type of coupling due to coagulation has not been modeled so far by any research team. Finally, during the RFA computation, the temperature at the tips of the probe is imposed. It would be more accurate to directly impose the delivered power as in [33] and thus simulate the cooling as well as the heating temperature distribution. This requires to solve the spatio-temporal electromagnetic heating problem, adding more complexity to the model, whose impact on the final thermal output is questionable and difficult to validate. Moreover, this raises some technical issues as Neumann instead of Dirichlet boundary condition would have to be applied.

\subsection{Effect of the Registration}

After several trials of registration algorithms, we were able to reliably estimate in some cases, the deformation between pre- and post-operative imaging to evaluate the necrosis extent prediction. But the registration method uses only the liver surface, and two lesions are poorly registered. However by taking the hepatic vessels into account as well, the registration could be improved. It should also be noted that the registration method differs from the one in [34], where the necrotic lesions were registered rigidly to the pre-operative image by aligning its barycenters which could introduce bias in the analysis. The impact of those registration processes has been evaluated on one tumor in this paper.

\subsection{Towards Personalization}

In clinical settings, due to the large variety of liver conditions found during RFA (cirrhosis, fibrosis, etc), we cannot assume inter-patient invariance of the biophysical parameters, as done here for the five pigs. Parameter personalization is then required and the proposed method may be suitable to get subject-specific parameters from easily accessible intra-operative quantities (the probe temperature and delivered power). We proposed a biophysical computational model whose computing time, although already shorter than the real physical process, remains too long to allow an easy use in a well established clinical workflow. But an extensive evaluation of such a comprehensive model is needed before simplifications are made to reduce its complexity and the invasive input data. The proposed method could then be translated into clinical practice, as several heating/cooling phases are generally necessary to perform a complete ablation. The first heating/cooling phase could be used to personalize the main parameters of the model, which could then give a patient-specific prediction of the lesion. Such a framework could help the clinician during the intervention: the probe position could be improved and/or the heating duration could be adjusted if the simulated lesion does not cover entirely the tumor for example. We evaluated the discrepancy in terms of temperature and delivered power, and we showed that key parameters can be estimated. This is a proof of concept not only for the ability to personalize the model but also for its predictive power by evaluating the simulation results on ten different tumors.

The necrotic extent is mainly controlled by the conductivity $\bar{d}_{t}$ and the heat capacity of vulnerable cells $c_{t}^{V}$ (not $\left.c_{t}^{U}\right) \cdot \bar{d}_{t}$ was not changed after optimization, so no significant difference in terms of necrotic area (mean of the point-to-mesh error: $6.0 \mathrm{~mm}$ versus $5.3 \mathrm{~mm}$ on average) or cooling temperature (error: $7.8^{\circ} \mathrm{C}$ versus $5.1^{\circ} \mathrm{C}$ ) appeared after personalization, despite a better match for the power (error: $20.4 \mathrm{~W}$ versus $25.9 \mathrm{~W}$ ) as illustrated on Fig. 16. Moreover, the point-to-mesh errors were of the order of $5 \mathrm{~mm}$; it suggests that simulations with optimized $\bar{d}_{t}$ and $c_{t}^{U}$ might be realistic in terms of necrotic area, power and temperature predictions.

The novel approach leads to an estimation of temperature away from the probe at any time during the ablation without requiring any information about the necrotic regions. This additional information could be used as surrogate to assess the amount and location of damaged tissue during the intervention (cells receiving excessive heat but without being necrosed) surrounding the ablated region. Furthermore, as the probe temperature and delivered power are available, in real-time, 
from the RF system, they could therefore be used for therapy guidance, provided that heat transfer can be computed much faster than real-time.

\subsection{Pre-clinical Study Challenges}

The pre-clinical validation approach was difficult to establish. It involves surrogate tumors implantation to mimic the RFA current clinical practice, however the surrogate tumors do not have any blood perfusion, and we assumed that they have tissue properties similar to hepatic tissue. It required acquisitions of different modalities (CT, MRI, US), at different times (pre-, intraand post-operative images). The different images at Day 1 (day of intervention) should be acquired quickly, as the anesthesia of the pig cannot last too long since the pig will be kept alive for 2 additional days. Due to the high complexity of the experimental set-up, preprocessing is necessary. The segmentation was performed semi-automatically, but the vessel meshes had to be smoothed to avoid unstable solution of the CFD solver. The probe was segmented from a CT image with a good resolution, but the artifacts induced by the probe and the bad resolution of the interventional MR images, did not allow to accurately register the probe position. The setting of subject-specific boundary conditions for the blood flow and pressure was not straightforward, since the acquisition process of Phase-Contrast MR images was complex to handle and requires a significant learning curve. Due to the non reproducible and noncoherent measures on the first four pigs, the measures of blood flows and pressures of Pig 5 only were used in all the computations.

Despite a comprehensive model and a complete preclinical study for its validation, many sources of errors are involved at the modeling level, but also at each preprocessing step. We are not able to accurately locate the position where the pressure was measured, but we assume that there is no variation in pressure in a small neighborhood of the two outlets. Even if the impact of the segmentation process has to be taken into account, we managed to identify most of the errors thanks to the pre-clinical study. In a clinical setup, the use of an invasive catheter to measure the pressure is not possible, neither the acquisition of Phase-Contrast MRI, intra-operative MRI with the probe, injected CT with the three different phases, which are not part of the standard clinical workflow for RFA. Even if many of the patient-specific parameters used in this study would not be precisely available for each patient, this work is a first step forward to get an insight on the most sensitive parameters to be personalized and those which do not affect the final output. A comprehensive biophysical model is therefore required for this evaluation. However, with a simplified blood flow model for example, those measurements will not be required. Mathematical models can improve the current clinical practice by providing more information to the clinician for the guidance but also for the planning of the procedure. However, the questions of validation and error in the predictions of such computational models are often neglected, whereas it is absolutely key to their potential clinical use. Having a model as detailed as possible is important to fully evaluate the effects of the different biophysical phenomena involved, and it is paramount to identify the sources of errors. A study of the errors is a necessary next step before using model-based therapy in clinical routine. Knowing how the pre-processing errors (due to segmentation, vessels smoothing, post-to-pre or probe registration) propagate to the final computational outcome would be of great interest. It would help to understand the modeling errors and to be more confident on the resulting ablation. The validation could be improved by acquiring images of better resolution, but this would impact the well-being of animals in the current framework.

\section{Conclusion}

In this paper, we presented a sophisticated pre-clinical set-up to validate a complete multi-physics model of RFA. This comprehensive validation was based on pre, intra-, post-operative images and device-based measurements. The RFA computational model relies on LBM and takes into account the main biophysical phenomena (heat transfer, cellular necrosis, hepatic blood flow). The advection effect of the porous circulation [11] is considered by including a CFD model, robust to image noise and anisotropy to compute the venous and arterial blood flow in the liver parenchyma. We also showed that parameter estimation is possible to reduce the bias introduced by the use of nominal parameters. The approach was successfully evaluated on twelve ablations from five swines, opening new opportunities for RFA planning and guidance.

Acknowledgements Part of this work was funded by Inria, Siemens Healthcare, IHU Strasbourg (SimulAB project) and by the European Research Council (ERC Advanced Grant MedYMA 2011-291080).

The authors are grateful to Gael Fourré, Franck Blindauer, Mourad Bouhadjar and Rodrigo Cararo at the IHU Strasbourg for their valuable assistance in performing the experimental procedures.

Compliance with ethical standards 
Conflict of interest The authors declare that they have no conflict of interest.

Ethical approval All procedures performed in studies involving animals were in accordance with the ethical standards of the institution or practice at which the studies were conducted.

Informed consent This articles does not contain patient data.

\section{References}

1. S.K. Hall, E.H. Ooi, S.J. Payne, International Journal of Hyperthermia 31(5), 538 (2015)

2. E.J. Patterson, C.H. Scudamore, D.A. Owen, A.G. Nagy, A.K. Buczkowski, Annals of surgery 227(4), 559 (1998)

3. J. Crezee, J. Lagendijk, Physics in Medicine and Biology 35(7), 905 (1990)

4. I.A. Chang, U.D. Nguyen, Biomedical engineering online 3(1), 27 (2004)

5. C. Schumann, C. Rieder, S. Haase, K. Teichert, P. Süss, P. Isfort, P. Bruners, T. Preusser, International journal of computer assisted radiology and surgery pp. 1-11 (2015)

6. Y. Jiang, S. Mulier, W. Chong, M. Diel Rambo, F. Chen, G. Marchal, Y. Ni, Int. J. Modelling, Identification and Control 9(3), 225 (2010)

7. I. Altrogge, T. Preusser, T. Kroger, S. Haase, T. Patz, R.M. Kirby, Int. J. for Uncertainty Quantification 2(3) (2012)

8. S. Payne, R. Flanagan, M. Pollari, T. Alhonnoro, C. Bost, D. O'Neill, T. Peng, P. Stiegler, Philos T Roy Soc A 369(1954), 4233 (2011)

9. C. Audigier, T. Mansi, H. Delingette, S. Rapaka, V. Mihalef, E. Boctor, M. Choti, A. Kamen, N. Ayache, D. Comaniciu, IEEE Transactions on Medical Imaging 34(7), $1576(2015)$

10. X. Chen, G.M. Saidel, J Biomech 131 (2009)

11. C. Audigier, T. Mansi, H. Delingette, S. Rapaka, V. Mihalef, D. Carnegie, E. Boctor, M. Choti, A. Kamen, D. Comaniciu, N. Ayache, in MICCAI Workshop ABDI (2014)

12. C. Rieder, T. Kroeger, C. Schumann, H.K. Hahn, Visualization and Computer Graphics, Trans. on 17(12), 1812 (2011)

13. B.B. Frericks, J.P. Ritz, T. Albrecht, S. Valdeig, A. Schenk, K.J. Wolf, K. Lehmann, Investigative radiology 43(4), 211 (2008)

14. H.H. Pennes, J Appl Physiol 85(1), 5 (1998)

15. L.O. Schwen, M. Krauss, C. Niederalt, F. Gremse, F. Kiessling, A. Schenk, T. Preusser, L. Kuepfer, PLoS Comput Biol 10(3), e1003499 (2014)

16. D. ONeill, T. Peng, P. Stiegler, U. Mayrhauser, S. Koestenbauer, K. Tscheliessnigg, S. Payne, Ann Biomed Eng 39, 570 (2011)

17. S.N. Goldberg, G.S. Gazelle, C.C. Compton, P.R. Mueller, K.K. Tanabe, Cancer 88(11), 2452 (2000)

18. A.C. Lardo, E.R. McVeigh, P. Jumrussirikul, R.D. Berger, H. Calkins, J. Lima, H.R. Halperin, Circulation 102(6), 698 (2000)

19. T. Dickfeld, R. Kato, M. Zviman, S. Nazarian, J. Dong, H. Ashikaga, A.C. Lardo, R.D. Berger, H. Calkins, H. Halperin, Heart Rhythm 4(2), 208 (2007)

20. L. Soler, S. Nicolau, P. Pessaux, D. Mutter, J. Marescaux, Hepatobiliary surgery and nutrition 3(2), 73 (2014)

21. M.A. Gulsun, H. Tek, in MICCAI Workshop CVII (2006)

22. C. Guetter, H. Xue, C. Chefd'Hotel, J. Guehring, in Biomedical Imaging: From Nano to Macro, 2011 IEEE International Symposium on (IEEE, 2011), pp. 590-593
23. T.L. Horng, W.L. Lin, C.T. Liauh, T.C. Shih, Medical physics 34(4), 1312 (2007)

24. F. Faure, C. Duriez, H. Delingette, J. Allard, B. Gilles, S. Marchesseau, H. Talbot, H. Courtecuisse, G. Bousquet, I. Peterlik, S. Cotin, in Soft Tissue Biomechanical Modeling for Computer Assisted Surgery (Springer, 2012), pp. 283321

25. P. Nithiarasu, K. Seetharamu, T. Sundararajan, International Journal of Heat and Mass Transfer 40(16), 3955 (1997)

26. Z. Guo, T. Zhao, Physical Review E 66(3), 036304 (2002)

27. S. Chen, G.D. Doolen, Annual review of fluid mechanics 30(1), 329 (1998)

28. C. Pan, L.S. Luo, C.T. Miller, Computers \& fluids $35(8)$, $898(2006)$

29. T. Peng, D. ONeill, S. Payne, International Journal of Heat and Mass Transfer 54(9), 2100 (2011)

30. M. Breen, X. Chen, D. Wilson, G. Saidel, in $E M B S / B M E S$ Conference, 2002., vol. 1 (IEEE, 2002), vol. 1, pp. 715-vol

31. T. Livraghi, S.N. Goldberg, S. Lazzaroni, F. Meloni, L. Solbiati, G.S. Gazelle, Radiology 210(3), 655 (1999)

32. Y. Zheng, A. Barbu, B. Georgescu, M. Scheuering, D. Comaniciu, in ICCV (IEEE, 2007), pp. 1-8

33. T. Kröger, I. Altrogge, T. Preusser, P.L. Pereira, D. Schmidt, A. Weihusen, H.O. Peitgen, in International Conference on Medical Image Computing and ComputerAssisted Intervention (Springer, 2006), pp. 380-388

34. C. Audigier, T. Mansi, H. Delingette, S. Rapaka, V. Mihalef, R. Pop, M. Diana, L. Soler, A. Kamen, D. Comaniciu, N. Ayache, in MICCAI Workshop on Computational Biomechanics for Medicine X (2015) 